\title{
Parental consanguinity in Hong Kong
}

\author{
KH Siong *, Sidney KC Au Yeung, TY Leung
}

\section{A B S T R A C T}

Introduction: Consanguineous union increases the risk of genetic disorders in offspring. The present study aimed to evaluate the prevalence and characteristics of parental consanguinity in Hong Kong, and its effects on pregnancy, perinatal, and child health outcomes.

Methods: Pregnant women in consanguineous unions attending an obstetrics unit at a public hospital in Hong Kong were retrospectively studied. Their pregnancy, perinatal, and child health outcomes were compared with an ethnicity-matched control group of pregnant women in non-consanguineous unions.

Results: The overall prevalence of parental consanguinity was $0.6 \%$ (first cousins or closer, $78.4 \%$; beyond first cousins, $21.6 \%$ ). The majority were ethnic Pakistani (85.0\%). Women in consanguineous unions were more likely to have an obstetric history of congenital abnormality (10.5\%), unexplained neonatal death $(4.6 \%)$, or family history of congenital abnormality (4.6\%). Offspring of unexplained intrauterine fetal demise (4.2\%) and

risk of recessive diseases (odds ratio $[\mathrm{OR}]=8.70$, $95 \%$ confidence interval $[\mathrm{CI}]=1.06-71.36)$, structural abnormalities $(\mathrm{OR}=4.55,95 \% \mathrm{CI}=2.17-9.53)$ and developmental delay $(\mathrm{OR}=6.72, \quad 95 \% \quad \mathrm{CI}=1.48$ $30.63)$, and significantly higher incidence of autistic spectrum disorder $(2.1 \% ; \mathrm{P}=0.008)$.

Conclusions: It is essential that information on the increased risks associated with parental consanguinity is included in genetic counselling for consanguineous couples, so that they can make informed decisions.

\section{Hong Kong Med J 2019;25:192-200}

https://doi.org/10.12809/hkmj187783

\footnotetext{
${ }^{1}$ KH Siong *, MB, BS, FHKAM (Obstetrics and Gynaecology)

${ }^{1}$ SKC Au Yeung, MB, BS, FRCOG

2 TY Leung, MD, FRCOG

1 Department of Obstetrics and Gynaecology, Tuen Mun Hospital, Tuen Mun, Hong Kong

2 Department of Obstetrics and Gynaecology, The Chinese University of Hong Kong, Prince of Wales Hospital, Shatin, Hong Kong
}

consanguineous parents had significantly higher * Corresponding author: skh664@ha.org.hk

New knowledge added by this study

- The majority of consanguineous unions in Hong Kong are of Pakistani ethnicity.

- It is well known that, in addition to recessive genetic diseases, offspring of consanguineous unions have higher incidences of non-genetically confirmed structural abnormalities, developmental delay, and autism spectrum disorders. The present study confirms this in the Hong Kong population.

Implications for clinical practice or policy

- Identification of consanguineous couples is essential to ensure appropriate referral for genetic counselling and diagnosis.

- Health education and information about availability of carrier screening should be provided for consanguineous couples to make informed choices.

\section{Introduction}

'Consanguinity' is a term derived from the Latin word 'consanguineus', meaning 'of the same blood'. In medical genetics, consanguineous union is generally referred as a union between couples related as second cousins or closer. ${ }^{1}$ The prevalence of consanguinity varies significantly worldwide, depending on cultural background, religious belief, and geography. The highest rates are estimated in the Near and Middle East and in Northern Africa, where $20 \%$ to $50 \%$ of marriages are consanguineous. ${ }^{1,2}$ The prevalence in Southern Europe, South America, and Japan is about $1 \%$ to $5 \%$, whereas Western European countries, North America, and Oceania have the lowest prevalence of $<1 \%$.,
Consanguineous union increases the risk of genetic disorders in offspring, especially for autosomal recessive diseases. However, recent studies suggest that parental consanguinity is also a risk factor for other adverse outcomes, even in developed multi-ethnic countries where the prevalence of consanguineous marriages is perceived as lower. For example, in Vienna where the background consanguinity rate was $<1 \%$, Posch et $\mathrm{al}^{3}$ reported that $39.7 \%$ of consanguineous couples had obstetric history of congenital malformations or genetic disorders. Becker et $\mathrm{al}^{4}$ reported that $6.1 \%$ of consanguineous couples were referred to a specialist centre in Germany for a history of major fetal anomalies. A 10-year retrospective analysis 
conducted in Australia, where the consanguinity rate is $5.5 \%$, concluded that parental consanguinity was associated with higher rates of threatened premature labour, fetal congenital abnormality, stillbirth, and perinatal mortality. ${ }^{5}$ In that study, consanguinity was also found to be an independent risk factor of nearly 3-fold for stillbirth.

In Hong Kong, parental consanguinity is more frequent among non-Chinese ethnic minorities, which account for $8 \%$ of the total population. ${ }^{6}$ Internationally, healthcare workers lack knowledge on the risks of consanguinity. ${ }^{7-9}$ Inconsistencies in information provided during genetic counselling and screening has been observed. ${ }^{10}$ Consanguineous couples are often unaware of the potential health hazards in their offspring. ${ }^{11-13}$ The level of concern and awareness of the adverse effects of parental consanguinity among patients and physicians is low, and available data on consanguinity in Hong Kong are limited. Therefore, in the present study, we aimed to clarify the prevalence and characteristics of pregnancies from consanguineous unions in Hong Kong, and to assess the related effects on maternal, perinatal, and child health outcomes.

\section{Methods}

The Prenatal Diagnosis Clinic in Tuen Mun Hospital is responsible for counselling consanguineous couples. Dating ultrasound and counselling sessions for Down syndrome screening are arranged for all pregnant women who have their booking appointment in our locality. At the booking appointment, patients are also asked about consanguinity. Hospital-accredited interpreters are arranged for couples who are not fluent in Cantonese or English. Identification of consanguineous cases depends on self-reporting by couples. A pedigree chart is constructed for each case. Couples are counselled about the possible effects of parental consanguinity on pregnancy outcomes, and advised to attend antenatal care regularly.

A retrospective cohort study of all parental

\section{在香港父母乃血親通婚的情況 常家雄、歐陽錦全、梁德楊}

引言：血親通婚會增加後裔患上遺傳基因毛病的風險。本研究旨在評 估香港現時血親通婚的普遍情況和特點，及其對懷乃期、後裔嬰孩及 兒童期健康的影響。

方法：在一所香港公立醫院產科部門進行對血親通婚之孕婦的回溯性 研究, 將她們的懷乃期、後裔嬰孩及兒童期健康結果與同一種族的非 血親通婚之孕婦的結果作比較。

結果：血親通婚的普及率為 $0.6 \%$ (堂表通婚或更近親通婚佔 $78.4 \%$; 堂表以外通婚佔 $21.6 \%$ ）。大部分血親通婚之孕婦為巴基斯坦裔 （85.0\%）。血親通婚之㝋婦較常有先天缺陷（10.5\%）、原因不明 的宮內胎兒死亡 $(4.2 \%)$ 、原因不明的新生兒死亡 (4.6\%) 的產科病 史, 以及有先天缺陷的家族病史 $(4.6 \%)$ 。血親通婚的後裔有明顯較 高患有隱性遺傳病（對比值：8.70，95\%置信區間：1.06-71.36）

結構缺陷（對比值：4.55，95\%置信區間：2.17-9.53）和發展遲緩 （對比值：6.72，置信區間：1.48-30.63）的風險, 自閉症譜系障礙 的患病率亦明顯較高 $(2.1 \% ; \mathrm{P}=0.008)$ 。

結論：在遣傳諮詢的過程中, 應對血親通婚夫婦提供他們後裔有較高 健康風險的資訊, 讓他們作出適合自己的檢查決定。

consanguinity cases over a 10 -year period from 1 January 2007 to 31 December 2016 was conducted. The antenatal records of these cases were reviewed. Details were gathered about pregnancy loss, fetal congenital abnormalities, pregnancy and perinatal outcomes, and neonatal and childhood development in the preceding pregnancy. The family history of each case was also collected from patient records, including known genetic or congenital anomalies, or intellectual or developmental disabilities. A morphology scan was arranged for consanguineous cases. Each family pedigree was studied to determine the degree of parental consanguinity (Fig). Only couples fulfilling the definition of consanguineous unions (second cousins or closer) were included for analysis in the present study.

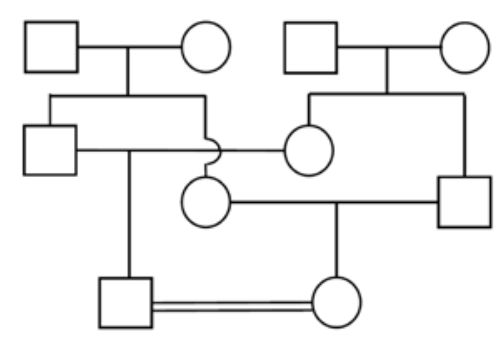

Double first cousins $\mathrm{F}=0.125$

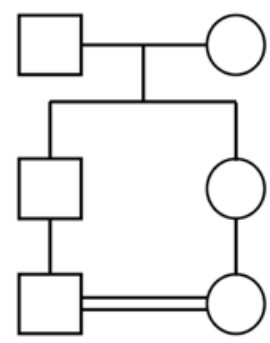

First cousins $\mathrm{F}=0.0625$

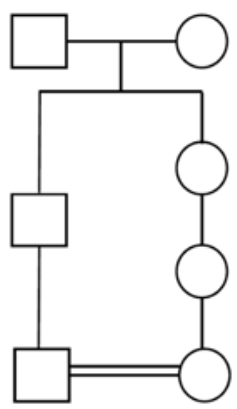

First cousins once removed $\mathrm{F}=0.0313$

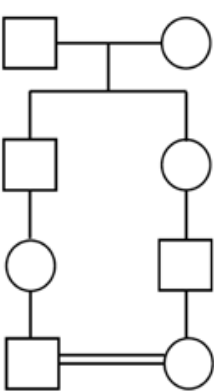

Second cousins $\mathrm{F}=0.0156$

FIG. Family trees of consanguineous marriages with corresponding coefficients of inbreeding (F) 
Socio-demographic characteristics were collected, including ethnicity, maternal and paternal age, religious beliefs, working status, education level, and occupation. Maternal antepartum and peripartum characteristics, and fetal and perinatal information were available. Information about the neonatal, infancy, and childhood outcomes of the offspring were retrieved from the public sector electronic record system.

The relationship between consanguinity and fetal, neonatal, infant, or childhood diseases that required long-term paediatric management was evaluated and categorised into one of three categories:

Category A-Improbable association with consanguinity: cases known to be caused by numerical or structural chromosomal abnormalities, or not to have an autosomal recessive mode of inheritance;

Category B-Probable association with consanguinity: cases known to have an autosomal recessive mode of inheritance, particularly when both parents were found to be the carriers of genetic disorders; and

Category C-Possible/unclear association with consanguinity: cases where the mode of inheritance was unclear, or when genetic testing was unremarkable.

The characteristics and outcomes of consanguineous cases were compared with a control group of non-consanguineous unions. The next record of a non-consanguineous case of the same ethnicity after that of a case of consanguineous union was selected as the control. This ensured the similar composition of ethnicity which might have socioeconomic effects on the maternal and fetal outcomes within the study and control groups. ${ }^{14}$ As some consanguineous couples might have contributed more than one pregnancies in our database, only adverse past obstetric outcome in the immediately preceding pregnancy was counted in the analysis, and any positive family history reported by such couples was counted as one case only, in order to prevent duplicated entries for multigravida women. Most previous studies have not evaluated the effects of closer consanguinity that might increase risks of hereditary disorders. ${ }^{5,15,16}$ To evaluate the effect of degree of inbreeding, comparisons were made among 'first cousin or closer' (including first cousin and double first cousin), 'beyond first cousin' (including first cousin once removed and second cousin), and non-consanguineous relationships.

Approval of this study was granted by the research and ethics committee of the study hospital. Guidelines for reporting observational studies according to the Strengthening the Reporting of Observational Studies in Epidemiology (STROBE) statement were followed.

Statistical analysis was performed using SPSS (Windows version 22.0; IBM Corp, Armonk [NY], US). Cross-tabulation between degrees of consanguinity and the different variables was performed in order to evaluate the characteristics of the study population. Differences in continuous variables were compared using $t$ test or one-way analysis of variance. Differences in categorical variables were analysed with Chi squared test or Fisher's exact test. Linear regression was carried out to adjust the collinearity among variables. Multivariate logistic regression analysis was used to determine the risk of consanguinity for adverse pregnancy and perinatal outcomes, with adjustment of significant confounders. Adjusted odds ratio (OR) with $95 \%$ confidence interval $(\mathrm{CI})$ were calculated. Statistical significance was established for $\mathrm{P}<0.05$.

\section{Results}

Of 56657 fetuses, 334 (0.6\%) were conceived by consanguineous parents; of these, the majority $(85.0 \%, 284$ of 334) were ethnic Pakistani (among whom the prevalence of consanguineous union is highest, at $30.5 \%)$, followed by Indian (6.2\%), Nepalese (2.7\%), Filipino (0.4\%), and Chinese $(0.04 \%)$ [Table 1]. Of all consanguineous unions, the majority were first cousin consanguineous unions $(76.6 \%)$

TABLE I. Ethnicity and consanguinity in mothers of 56657 fetuses from 2007 to 2016

\begin{tabular}{|c|c|c|c|c|c|c|}
\hline \multirow[t]{2}{*}{ Ethnicity } & \multirow[t]{2}{*}{ No. of fetuses } & \multicolumn{2}{|c|}{$\begin{array}{l}\text { First cousin or closer }(n=262 \text {, } \\
\qquad 78.4 \%)\end{array}$} & \multicolumn{2}{|c|}{ Beyond first cousin $(n=72,21.6 \%)$} & \multirow{2}{*}{$\begin{array}{l}\text { Total parental } \\
\text { consanguinity in } \\
\text { ethnicity }\end{array}$} \\
\hline & & First cousin & $\begin{array}{l}\text { Double first } \\
\text { cousin }\end{array}$ & $\begin{array}{l}\text { First cousin } \\
\text { once removed }\end{array}$ & Second cousin & \\
\hline All ethnicities & 56657 & $256(76.6 \%)$ & $6(1.8 \%)$ & $17(5.1 \%)$ & $55(16.5 \%)$ & $334(0.6 \%)$ \\
\hline Chinese & 49032 & 17 & 0 & 0 & 3 & $20(0.04 \%)$ \\
\hline Filipino & 235 & 0 & 0 & 0 & 1 & $1(0.4 \%)$ \\
\hline Indian & 177 & 11 & 0 & 0 & 0 & $11(6.2 \%)$ \\
\hline Nepalese & 669 & 13 & 0 & 0 & 5 & $18(2.7 \%)$ \\
\hline Pakistani & 931 & 215 & 6 & 17 & 46 & $284(30.5 \%)$ \\
\hline Others & 5613 & 0 & 0 & 0 & 0 & 0 \\
\hline
\end{tabular}


and double first cousin unions (1.8\%); together, including maternal and paternal age, education level, these were categorised as first cousin or closer $(\leq 1 C)$ religion, length of stay in Hong Kong, marital status, unions. The remainder were categorised as beyond working status, occupation, parity, and body mass first cousin $(>1 C)$ unions, and included first cousin index showed no significant differences between the once removed unions (5.1\%), and second cousin consanguineous group and the non-consanguineous unions (16.5\%). Comparison of background variables control group (Table 2).

TABLE 2. Background characteristics of 334 fetuses with consanguineous parents and 334 control fetuses with nonconsanguineous parents*

\begin{tabular}{|c|c|c|c|}
\hline Characteristics & $\begin{array}{l}\text { Non-consanguineous } \\
\text { parents }(n=334)\end{array}$ & $\begin{array}{l}\text { Consanguineous parents } \\
\qquad(n=334)\end{array}$ & $P$ value \\
\hline Maternal age (years) & $28.4 \pm 5.25$ & $28.3 \pm 5.37$ & 0.776 \\
\hline$<20$ & $16(4.8 \%)$ & $12(3.6 \%)$ & 0.638 \\
\hline $20-35$ & $282(84.4 \%)$ & $281(84.1 \%)$ & \\
\hline$\geq 35$ & $36(10.8 \%)$ & $41(12.3 \%)$ & \\
\hline \multicolumn{4}{|l|}{ Maternal education level } \\
\hline Elementary & $105(31.4 \%)$ & $117(35.0 \%)$ & 0.600 \\
\hline Secondary & $171(51.2 \%)$ & $164(49.1 \%)$ & \\
\hline Tertiary & $58(17.4 \%)$ & $53(15.9 \%)$ & \\
\hline \multicolumn{4}{|l|}{ Maternal religion } \\
\hline Buddhist & $18(5.4 \%)$ & $18(5.3 \%)$ & 0.192 \\
\hline Christian & $3(0.9 \%)$ & $1(0.3 \%)$ & \\
\hline Hindu & $14(4.2 \%)$ & $9(2.7 \%)$ & \\
\hline Muslim & 277 (82.9\%) & $276(82.6 \%)$ & \\
\hline Nil & $10(3.0 \%)$ & $3(0.9 \%)$ & \\
\hline Not reported & $12(3.6 \%)$ & $27(8.2 \%)$ & \\
\hline Maternal length of stay in Hong Kong (years) & $8.89 \pm 8.88$ & $8.69 \pm 8.79$ & 0.772 \\
\hline Married & $334(100 \%)$ & $334(100 \%)$ & - \\
\hline Working mother & 35 (10.5\%) & $28(8.4 \%)$ & 0.354 \\
\hline Paternal age (years) & $33.0 \pm 6.54$ & $31.6 \pm 5.52$ & 0.070 \\
\hline \multicolumn{4}{|l|}{ Paternal occupation } \\
\hline Unskilled manual & 77 (23.1\%) & $116(34.7 \%)$ & 0.810 \\
\hline Skilled manual/professional & $82(24.6 \%)$ & $119(35.6 \%)$ & \\
\hline Self-employed & $43(12.8 \%)$ & $51(15.3 \%)$ & \\
\hline Unemployed & $23(6.9 \%)$ & $34(10.2 \%)$ & \\
\hline Not reported & 109 (32.6\%) & $14(4.2 \%)$ & \\
\hline \multicolumn{4}{|l|}{ Parity } \\
\hline 0 & $83(24.9 \%)$ & $95(28.4 \%)$ & 0.248 \\
\hline $1-2$ & $169(50.6 \%)$ & $153(45.9 \%)$ & \\
\hline$\geq 3$ & $82(24.5 \%)$ & $86(25.7 \%)$ & \\
\hline \multicolumn{4}{|l|}{ Maternal body mass index $\left(\mathrm{kg} / \mathrm{m}^{2}\right)$} \\
\hline$<18.5$ & $19(5.7 \%)$ & $21(6.3 \%)$ & 0.727 \\
\hline 18.5 to $<25$ & $146(43.7 \%)$ & $128(38.3 \%)$ & \\
\hline 25 to $<30$ & $103(30.8 \%)$ & $114(34.1 \%)$ & \\
\hline 30 to $<35$ & $47(14.1 \%)$ & $54(16.2 \%)$ & \\
\hline 35 to $<40$ & $14(4.2 \%)$ & $14(4.2 \%)$ & \\
\hline$\geq 40$ & $5(1.5 \%)$ & $3(0.9 \%)$ & \\
\hline Spontaneous conception & $334(100 \%)$ & $334(100 \%)$ & - \\
\hline Maternal smoking/substance abuse & 0 & 0 & - \\
\hline
\end{tabular}

* Data are shown as mean \pm standard deviation or No. (\%), unless otherwise specified 
Women in consanguineous unions were significantly more likely to have experienced congenital abnormality $(10.5 \%$ vs $0.4 \% ; \mathrm{P}<0.001)$, unexplained intrauterine fetal demise $(4.2 \%$ vs $0.4 \%$; $\mathrm{P}=0.005)$ and neonatal death $(4.6 \%$ vs $1.2 \% ; \mathrm{P}=0.024)$ in the preceding pregnancy, and family history of congenital abnormality ( $4.6 \%$ vs $0 \%$; $\mathrm{P}<0.001)$ than were non-consanguineous controls (Table 3). Down syndrome screening was offered to all women, but the attendance was only about one-fifth for all groups.

In terms of major maternal and perinatal complications, there were no significant differences between the non-consanguineous control group and the overall consanguineous group or the subgroups, except that pregnancies of $\leq 1 \mathrm{C}$ unions were more often complicated with pre-eclampsia ( $4.2 \%$ vs $1.2 \%$; $\mathrm{P}=0.02)$ than were those of the non-consanguineous control group (Table 4).

Altogether there were 58 fetuses and 14 fetuses having different abnormalities, from 55 consanguineous and 14 control couples respectively (Table 5). Offspring of consanguineous couples had a higher risk of having category $\mathrm{C}$ disorders $(\mathrm{OR}=4.60$; $95 \% \mathrm{CI}=2.35-9.00)$ or category $\mathrm{B}$ disorders $(\mathrm{OR}=8.70$;
95\% CI=1.06-71.36), compared with those of nonconsanguineous couples. The overall prevalence of category C disorders (14.7\%) was higher than that of category B disorders (2.4\%). Compared with the non-consanguineous control group, the prevalence of category $\mathrm{C}$ disorders was significantly higher in the $\leq 1 \mathrm{C}$ subgroup $(\mathrm{OR}=5.59$; $95 \% \mathrm{CI}=2.83-11.06)$; it was lower in the $>1 \mathrm{C}$ subgroup, but the difference was not significant.

The prevalence of structural malformations was higher in the consanguineous group than that in the non-consanguineous control group, especially for those abnormalities involving cardiovascular, musculoskeletal, and urological systems (Table 5). Parental consanguinity also significantly increased the risk of developmental delay in offspring of consanguineous couples $(\mathrm{OR}=6.72,95 \% \mathrm{CI}=1.48$ $30.63)$ and in those of $\leq 1 \mathrm{C}$ couples $(\mathrm{OR}=7.64,95 \%$ $\mathrm{CI}=1.64-35.58)$. Autism spectrum disorder was more prevalent in offspring of consanguineous couples (2.1\%) than in those of non-consanguineous couples $(0 \%)[\mathrm{P}=0.008]$. The diseases recorded in the consanguineous group and in the control group are detailed in online supplementary Appendices 1 and 2 , respectively.

TABLE 3. Pregnancy characteristics of 334 fetuses with consanguineous parents and 334 control fetuses with non-consanguineous parents*

\begin{tabular}{|c|c|c|c|c|c|c|c|}
\hline Characteristics & $\begin{array}{c}\text { Non- } \\
\text { consanguineous } \\
\text { parents }(n=334)\end{array}$ & $\begin{array}{l}\text { Consanguineous } \\
\text { parents }(n=334)\end{array}$ & $P$ value & $\begin{array}{l}\text { First cousin } \\
\text { or closer } \\
(n=262)\end{array}$ & P valuet & $\begin{array}{c}\text { Beyond } \\
\text { first cousin } \\
(n=72)\end{array}$ & $P$ value \\
\hline $\begin{array}{l}\text { T1 miscarriage in the preceding } \\
\text { pregnancy }\end{array}$ & $72(27.1 \%) \ddagger$ & $82(32.7 \%) \ddagger$ & 0.164 & $71(35.5 \%) \ddagger$ & 0.051 & $11(21.6 \%) \ddagger$ & 0.431 \\
\hline $\begin{array}{l}\text { T2 miscarriage in the preceding } \\
\text { pregnancy }\end{array}$ & $2(0.8 \%) \ddagger$ & $6(2.4 \%) \ddagger$ & 0.131 & $5(2.5 \%) \ddagger$ & 0.125 & $1(2.0 \%) \ddagger$ & 0.414 \\
\hline $\begin{array}{l}\text { Preceding child with congenital } \\
\text { abnormality }\end{array}$ & $1(0.4 \%) \S$ & $25(10.5 \%) \S$ & $<0.001$ & $22(11.6 \%) \S$ & $<0.001$ & $3(6.1 \%) \S$ & 0.001 \\
\hline Preceding child with ID/DD & $2(0.8 \%) \S$ & $6(2.5 \%) \S$ & 0.135 & $6(3.2 \%) \S$ & 0.066 & $0 \S$ & 0.531 \\
\hline $\begin{array}{l}\text { Unexplained IUFD in the preceding } \\
\text { pregnancy }\end{array}$ & $1(0.4 \%) \S$ & $10(4.2 \%) \S$ & 0.005 & $9(4.7 \%) \S$ & 0.002 & $1(2.0 \%) \S$ & 0.196 \\
\hline $\begin{array}{l}\text { Unexplained NND in the preceding } \\
\text { pregnancy }\end{array}$ & $3(1.2 \%) \S$ & $11(4.6 \%) \S$ & 0.024 & $11(5.8 \%) \S$ & 0.006 & $0 \S$ & 0.442 \\
\hline Family history of congenital abnormality & 0 & $9(4.6 \%) \|$ & $<0.001$ & $5(3.3 \%) \|$ & 0.001 & $4(9.1 \%) \|$ & $<0.001$ \\
\hline Family history of ID/DD & 0 & $2(1.0 \%) \|$ & 0.064 & $1(0.7 \%) \|$ & 0.138 & $1(2.3 \%) \|$ & 0.005 \\
\hline Multiple pregnancy & $4(1.2 \%)$ & $6(1.8 \%)$ & 0.524 & $4(1.5 \%)$ & 0.729 & $2(2.8 \%)$ & 0.314 \\
\hline DSS performed & $78(23.4 \%)$ & $65(19.5 \%)$ & 0.220 & $52(19.8 \%)$ & 0.304 & $13(18.1 \%)$ & 0.328 \\
\hline Structural scan performed & 56 (16.8\%) & $192(57.5 \%)$ & $<0.001$ & $153(58.4 \%)$ & $<0.001$ & $39(54.2 \%)$ & $<0.001$ \\
\hline
\end{tabular}

Abbreviations: DSS = Down syndrome screening; ID/DD = intellectual disability or developmental delay; IUFD = intrauterine fetal demise; NND = neonatal death; $\mathrm{TI}$ = first trimester; T2 = second trimester

* Data are shown as No. (\%), unless otherwise specified

† Comparison with non-consanguineous controls

‡ Primigravida women excluded in the calculation, only multigravida women used for analysis (non-consanguineous, 266; overall consanguineous, 25 I; first cousin or closer, 200; beyond first cousin, 51)

$\S$ Nulliparous women excluded in the calculation, multiparous women used for analysis (non-consanguineous, 25 I; overall consanguineous, 239 ; first cousin or closer, 190; beyond first cousin, 49)

॥ Positive family history reported by a multigravida woman was counted as one case only to avoid duplicated data entries (non-consanguineous, 334; overall consanguineous, 196; first cousin or closer, 152; beyond first cousin, 44) 
TABLE 4. Maternal and perinatal outcomes of 334 fetuses with consanguineous parents and 334 control fetuses with non-consanguineous parents*

\begin{tabular}{|c|c|c|c|c|c|c|c|}
\hline Outcomes & $\begin{array}{c}\text { Non- } \\
\text { consanguineous } \\
\text { parents }(n=334)\end{array}$ & $\begin{array}{l}\text { Consanguineous } \\
\text { parents }(n=334)\end{array}$ & $P$ value & $\begin{array}{l}\text { First cousin or } \\
\text { closer }(n=262)\end{array}$ & $\mathrm{P}$ valuet & $\begin{array}{l}\text { Beyond first } \\
\text { cousin }(n=72)\end{array}$ & $P$ value \\
\hline Diabetes & $46(13.8 \%)$ & $59(17.7 \%)$ & 0.167 & $51(19.5 \%)$ & 0.062 & $8(11.1 \%)$ & 0.546 \\
\hline Hypertensive disorders & $20(6.0 \%)$ & $17(5.1 \%)$ & 0.612 & $11(4.2 \%)$ & 0.329 & $6(8.3 \%)$ & 0.461 \\
\hline Pre-eclampsia & $4(1.2 \%)$ & $11(3.3 \%)$ & 0.068 & $11(4.2 \%)$ & 0.020 & 0 & 0.351 \\
\hline $\mathrm{APH}$ & $8(2.4 \%)$ & $14(4.2 \%)$ & 0.193 & $10(3.8 \%)$ & 0.314 & $4(5.6 \%)$ & 0.145 \\
\hline TOP & 0 & $2(0.6 \%)$ & 0.157 & $2(0.8 \%)$ & 0.110 & 0 & - \\
\hline Miscarriage & 0 & $1(0.3 \%)$ & 0.317 & $1(0.4 \%)$ & 0.258 & 0 & - \\
\hline IUFD & $2(0.6 \%)$ & $1(0.3 \%)$ & 0.563 & $1(0.4 \%)$ & 0.710 & 0 & 0.510 \\
\hline Preterm labour & $32(9.6 \%)$ & $36(10.8 \%)$ & 0.609 & $27(10.3 \%)$ & 0.769 & $9(12.5 \%)$ & 0.456 \\
\hline GA at delivery (weeks) & $38.5 \pm 2.31$ & $38.5 \pm 1.94$ & 0.791 & $38.4 \pm 2.02$ & 0.766 & $38.5 \pm 1.63$ & 0.978 \\
\hline Spontaneous labour & 185 (55.4\%) & $163(48.8 \%)$ & 0.88 & 127 (48.5\%) & 0.093 & $36(50 \%)$ & 0.405 \\
\hline \multicolumn{8}{|l|}{ MOD } \\
\hline Normal VD & 208 (62.3\%) & 199 (59.6\%) & 0.493 & 157 (59.9\%) & 0.707 & 42 (58.3\%) & 0.376 \\
\hline Instrumental VD & $19(5.7 \%)$ & $15(4.5 \%)$ & & $13(5.0 \%)$ & & $2(2.8 \%)$ & \\
\hline Caesarean section & $107(32.0 \%)$ & $120(35.9 \%)$ & & $92(35.1 \%)$ & & 28 (38.9\%) & \\
\hline PPH & $14(4.2 \%)$ & $13(3.9 \%)$ & 0.844 & $10(3.8 \%)$ & 0.817 & $3(4.2 \%)$ & 0.992 \\
\hline Birth weight (kg) & $3.11 \pm 0.59$ & $3.07 \pm 0.54$ & 0.365 & $3.07 \pm 0.55$ & 0.409 & $3.07 \pm 0.47$ & 0.584 \\
\hline IUGR/SGA baby & $24(7.2 \%)$ & 33 (9.9\%) & 0.213 & $28(10.7 \%)$ & 0.133 & $5(6.9 \%)$ & 0.943 \\
\hline \multicolumn{8}{|l|}{ Sex } \\
\hline Female & $160(47.9 \%)$ & $163(48.8 \%)$ & 0.816 & $122(46.6 \%)$ & 0.745 & 41 (56.9\%) & 0.164 \\
\hline Male & $174(52.1 \%)$ & $171(51.2 \%)$ & & 140 (53.4\%) & & $31(43.1 \%)$ & \\
\hline AS $<7$ at 1 minute & $15(4.5 \%)$ & $10(3.0 \%)$ & 0.308 & $9(3.4 \%)$ & 0.515 & $1(1.4 \%)$ & 0.220 \\
\hline AS $<7$ at 5 minutes & $2(0.6 \%)$ & 0 & 0.157 & 0 & 0.210 & 0 & 0.510 \\
\hline NICU admission & $9(2.7 \%)$ & $16(4.8 \%)$ & 0.154 & $12(4.6 \%)$ & 0.215 & $4(5.6 \%)$ & 0.211 \\
\hline Neonatal sepsis & $7(2.1 \%)$ & $7(2.1 \%)$ & 1.000 & $7(2.7 \%)$ & 0.645 & 0 & 0.215 \\
\hline Neonatal RDS & $7(2.1 \%)$ & $8(2.4 \%)$ & 0.794 & $6(2.3 \%)$ & 0.872 & $2(2.8 \%)$ & 0.721 \\
\hline Neonatal jaundice & $29(8.7 \%)$ & $42(12.6 \%)$ & 0.103 & 35 (13.4\%) & 0.067 & 7 (9.7\%) & 0.778 \\
\hline Neonatal death & $3(0.9 \%)$ & $5(1.5 \%)$ & 0.477 & $3(1.1 \%)$ & 0.764 & $2(2.8 \%)$ & 0.190 \\
\hline
\end{tabular}

Abbreviations: $\mathrm{APH}=$ antepartum haemorrhage; $\mathrm{AS}=$ Apgar score; GA = gestational age; IUFD = intrauterine fetal demise; IUGR = intrauterine growth restriction; $\mathrm{MOD}=$ mode of delivery; $\mathrm{NICU}=$ neonatal intensive care unit; $\mathrm{PPH}=$ postpartum haemorrhage; $\mathrm{RDS}=$ respiratory distress syndrome; $\mathrm{SGA}=$ small for gestational age; $\mathrm{TOP}=$ termination of pregnancy; $\mathrm{VD}=$ vaginal delivery

* Data are shown as No. (\%) or mean \pm standard deviation, unless otherwise specified

$\dagger$ Comparison with non-consanguineous controls

\section{Discussion}

To the best of our knowledge, this is the first comprehensive study in Hong Kong describing the prevalence of parental consanguinity. Our results support those of previous studies that revealed a higher prevalence of parental consanguinity in certain ethnic groups, and the higher prevalence of known genetic disorders (category B) among their offspring. In addition, our study has revealed that the prevalence of fetal structural abnormalities, developmental delay, and autism spectrum disorders (category C) are also high. This has implications for prenatal counselling and diagnosis, and related healthcare services.

Our comparison of maternal age and parity showed no significant difference between the consanguineous group and control group. This is in contrast to findings by Islam et $\mathrm{al}^{16}$ and Hosseini-Chavoshi et al, ${ }^{17}$ who found that women in consanguineous unions were younger and of higher parity in Iran and Oman, where the consanguinity rate was more than $30 \%$. Studies in India and Pakistan populations also showed that mothers in consanguineous relationships were more likely to be socially and economically disadvantaged. ${ }^{11,18}$ The similarity in the socio-economic characteristics 
TABLE 5. Causative association of abnormalities with degree of parental consanguinity in 334 fetuses with consanguineous parents and 334 control fetuses with non-consanguineous parents

\begin{tabular}{|c|c|c|c|c|c|c|c|c|c|c|}
\hline Abnormalities & $\begin{array}{c}\text { Non- } \\
\text { consan- } \\
\text { guineous } \\
\text { parents } \\
(n=334)\end{array}$ & $\begin{array}{l}\text { Consan- } \\
\text { guineous } \\
\text { parents } \\
(n=334)\end{array}$ & $\begin{array}{l}\text { OR }(95 \% \\
\text { Cl) }\end{array}$ & $P$ value & $\begin{array}{c}\text { First } \\
\text { cousin } \\
\text { or closer } \\
(n=262)\end{array}$ & $\begin{array}{l}\mathrm{OR}^{\star}(95 \% \\
\mathrm{Cl})\end{array}$ & $P$ value & $\begin{array}{l}\text { Beyond } \\
\text { first } \\
\text { cousin } \\
(n=72)\end{array}$ & $\begin{array}{l}\mathrm{OR}^{\star}(95 \% \\
\mathrm{Cl})\end{array}$ & $P$ value \\
\hline $\begin{array}{l}\text { Category A: } \\
\text { Improbable } \\
\text { association with } \\
\text { consanguinity }\end{array}$ & $1(0.3 \%)$ & $1(0.3 \%)$ & $\begin{array}{c}1.31 \\
(0.08-21.13)\end{array}$ & 0.847 & $1(0.4 \%)$ & $\begin{array}{c}1.79 \\
(0.11-28.86)\end{array}$ & 0.681 & 0 & $<0.001$ & 0.997 \\
\hline $\begin{array}{l}\text { Category B: Probable } \\
\text { association with } \\
\text { consanguinity }\end{array}$ & $1(0.3 \%)$ & $8(2.4 \%)$ & $\begin{array}{c}8.70 \\
(1.06-71.36)\end{array}$ & 0.044 & $7(2.7 \%)$ & $\begin{array}{c}9.89 \\
(1.18-83.01)\end{array}$ & 0.035 & $1(1.4 \%)$ & $\begin{array}{c}5.43 \\
(0.33-89.18)\end{array}$ & 0.236 \\
\hline $\begin{array}{l}\text { Category C: Possible/ } \\
\text { unclear association } \\
\text { with consanguinity† }\end{array}$ & $12(3.6 \%)$ & 49 (14.7\%) & $\begin{array}{c}4.60 \\
(2.35-9.00)\end{array}$ & $<0.001$ & $45(17.2 \%)$ & $\begin{array}{c}5.59 \\
(2.83-11.06)\end{array}$ & $<0.001$ & $4(5.6 \%)$ & $\begin{array}{c}1.77 \\
(0.54-5.80)\end{array}$ & 0.348 \\
\hline Isolated involvementł & $12(3.6 \%)$ & $41(12.3 \%)$ & $\begin{array}{c}3.70 \\
(1.86-7.33)\end{array}$ & $<0.001$ & $38(14.5 \%)$ & $\begin{array}{c}4.44 \\
(2.21-8.90)\end{array}$ & $<0.001$ & $3(4.2 \%)$ & $\begin{array}{c}1.42 \\
(0.38-5.28)\end{array}$ & 0.605 \\
\hline Multiple involvement $\ddagger$ & 0 & $8(2.4 \%)$ & & $0.004 \S$ & $7(2.7 \%)$ & & $0.003 \S$ & $1(1.4 \%)$ & & $0.027 \S$ \\
\hline $\begin{array}{l}\text { Structural } \\
\text { abnormalities } \dagger\end{array}$ & $10(3.0 \%)$ & $38(11.4 \%)$ & $\begin{array}{c}4.55 \\
(2.17-9.53)\end{array}$ & $<0.001$ & $35(13.4 \%)$ & $\begin{array}{c}5.49 \\
(2.59-11.62)\end{array}$ & $<0.001$ & $3(4.2 \%)$ & $\begin{array}{c}1.73 \\
(0.45-6.65)\end{array}$ & 0.426 \\
\hline Cardiovascular & $7(2.1 \%)$ & $19(5.7 \%)$ & $\begin{array}{c}3.05 \\
(1.22-7.61)\end{array}$ & 0.017 & $17(6.5 \%)$ & $\begin{array}{c}3.55 \\
(1.40-9.04)\end{array}$ & 0.008 & $2(2.8 \%)$ & $\begin{array}{c}1.72 \\
(0.34-8.78)\end{array}$ & 0.514 \\
\hline Craniofacial & $2(0.6 \%)$ & $7(2.1 \%)$ & $\begin{array}{c}3.10 \\
(0.60-16.03)\end{array}$ & 0.178 & $6(2.3 \%)$ & $\begin{array}{c}3.84 \\
(0.72-20.52)\end{array}$ & 0.116 & $1(1.4 \%)$ & $\begin{array}{c}1.23 \\
(0.08-18.80)\end{array}$ & 0.883 \\
\hline Musculoskeletal & $1(0.3 \%)$ & $9(2.7 \%)$ & $\begin{array}{c}9.53 \\
(1.10-82.34)\end{array}$ & 0.040 & 9 (3.4\%) & $\begin{array}{c}12.48 \\
(1.42-110.03)\end{array}$ & 0.023 & 0 & 0.00 & 0.997 \\
\hline Gastrointestinal & 0 & $2(0.6 \%)$ & & $0.157 \S$ & $2(0.8 \%)$ & & $0.108 \S$ & 0 & - & \\
\hline Urological & 0 & $6(1.8 \%)$ & & $0.014 \S$ & $6(2.3 \%)$ & & $0.005 \S$ & 0 & - & \\
\hline $\begin{array}{l}\text { Non-syndromic } \\
\text { sensorineural } \\
\text { hearing loss }\end{array}$ & 0 & $3(0.9 \%)$ & & $0.083 \S$ & $3(1.1 \%)$ & & $0.050 \S$ & 0 & - & \\
\hline $\begin{array}{l}\text { Developmental and } \\
\text { behavioural disorders } \dagger\end{array}$ & 2 (0.6\%) & $14(4.2 \%)$ & $\begin{array}{c}6.72 \\
(1.48-30.63)\end{array}$ & 0.014 & 12 (4.6\%) & $\begin{array}{c}7.64 \\
(1.64-35.58)\end{array}$ & 0.001 & $2(2.8 \%)$ & $\begin{array}{c}4.11 \\
(0.53-31.91)\end{array}$ & 0.178 \\
\hline $\begin{array}{l}\text { Developmental } \\
\text { delay }\end{array}$ & $2(0.6 \%)$ & $14(4.2 \%)$ & $\begin{array}{c}6.72 \\
(1.48-30.63)\end{array}$ & 0.014 & 12 (4.6\%) & $\begin{array}{c}7.64 \\
(1.64-35.58)\end{array}$ & 0.001 & $2(2.8 \%)$ & $\begin{array}{c}4.11 \\
(0.53-31.91)\end{array}$ & 0.178 \\
\hline $\begin{array}{l}\text { Autism spectrum } \\
\text { disorder }\end{array}$ & 0 & 7 (2.1\%) & & $0.008 \S$ & $6(2.3 \%)$ & & $0.005 \S$ & $1(1.4 \%)$ & & $0.027 \S$ \\
\hline $\begin{array}{l}\text { Attention deficit } \\
\text { hyperactivity } \\
\text { disorder }\end{array}$ & 0 & $2(0.6 \%)$ & & $0.157 \S$ & 2 (0.8\%) & & $0.108 \S$ & 0 & - & \\
\hline
\end{tabular}

Abbreviations: $95 \% \mathrm{Cl}=$ confidence interval; $\mathrm{OR}=$ odds ratio

* Comparison with non-consanguineous controls

† A case may have clinical manifestations in more than one subcategory

‡ Subcategories used for analysis include cardiovascular, craniofacial, musculoskeletal, gastrointestinal, urological, developmental and behavioural, and hearing disorders

$\S$ No cases in the control group, Chi squared test is used instead to compare groups

between the consanguineous and non- supplementary Appendix 1). Although the carrier consanguineous unions of our study indicates that status of thalassaemia can be screened by low socio-economic factors are unlikely to be causes mean corpuscular volume of red blood cells, the of the poorer fetal outcomes, both in the index carrier status of other recessive disorders can be pregnancy and the preceding pregnancy, found in more complex. For some disorders, comprehensive our consanguineous group.

We identified eight offspring with autosomal required., ${ }^{419-21}$ Our data provide useful information recessive diseases in the consanguineous group, for preconception counselling for consanguineous including three cases of beta-thalassaemia major couples. However, exome sequencing is expensive, and five cases of other rarer diseases (online and this screening test is not yet available in public 
hospitals. Health education and information about the availability of carrier screening should be provided to all pregnant women, regardless of cultural, religious, or socio-economic background. Once a consanguineous couple is diagnosed to be the carrier of a genetic disease, they should be encouraged to discuss carrier screening with their siblings, who may also carry the same recessive gene and be in consanguineous union. Access to obstetric care and genetic counselling services in prenatal diagnosis clinics allows couples to make informed choices. Knowledge on various cultural, religious, or socio-economic issues allows healthcare workers to provide appropriate support and to best advise patients.

Our results revealed that category $C$ disorders are more prevalent among offspring of consanguineous couples, especially in the $\leq 1 \mathrm{C}$ subgroup. Fetal structural ultrasonographic examination should be offered to $\leq 1 \mathrm{C}$ couples, especially for the cardiovascular, urological, and skeletal systems. ${ }^{22-26}$ Detailed genetic counselling and investigation services must be offered to $\leq 1 \mathrm{C}$ couples if fetal abnormalities are detected. ${ }^{3,4}$

Our results revealed increased risk of developmental and behavioural disorders for offspring of consanguineous couples. However, disorders such as developmental delay and autism spectrum disorder are not diagnosable before birth. Preconception and prenatal counselling should be offered to consanguineous couples, who should also be reminded about regular postnatal followup examinations, in order to avoid any delay in diagnosing any developmental or behavioural disorders. ${ }^{27}$

Pakistani ethnicity accounted for only $1.6 \%$ of all fetuses but $85 \%$ of consanguineous couples in our study. According to the Hong Kong 2016 population by-census, $0.25 \%$ of the total Hong Kong population was of Pakistani ethnicity. ${ }^{6}$ However, the majority of this local Pakistani population is within potentially reproductive age-groups (15-24 years, 19.2\%; 25-34 years, $14.9 \%$; $35-44$ years, $21.3 \%$ ), and they tend to have more children per couple than do ethnic Chinese couples. ${ }^{6}$ It is essential to include information about the increased risks of parental consanguinity during the antenatal care and provide appropriate genetic counselling once a consanguineous couple is identified.

In addition to poor fetal outcomes, we also found a 3-fold increased risk of pre-eclampsia among women in $\leq 1 \mathrm{C}$ unions. Familial aggregation and possible genetic correlation of pre-eclampsia have been observed, but the exact effect of consanguinity remains controversial. ${ }^{28,29}$ Mumtaz et $\mathrm{al}^{15}$ suggested that parental consanguinity is a risk factor of 1.6fold for preterm birth at less than 33 weeks of gestation. Low birth weight has also been associated with first-cousin relationships, but the risk increase was found to be marginal $(\mathrm{OR}=1.36)^{30}$. Our study did not confirm higher incidences of antepartum, peripartum, neonatal and perinatal complications in overall consanguinity. Findings on the effect of consanguinity on various complications are inconsistent, especially when these complications are multifactorial in pathogenesis. . $15,27,29,30^{2}$

One limitation of our study is the retrospective nature that might have led to incompleteness of information for analysis, especially when previous pregnancies were not in Hong Kong. Another limitation is that some of the fetal abnormalities classified under category $\mathrm{C}$ may in fact be category $B$ disorders, as some of them recurred in the same couples (online supplementary Appendix 1); the majority of category $\mathrm{C}$ disorders did not receive genetic investigations. However, there is a high dependence on public health service in our locality, and this facilitated data retrieval of postnatal, infancy, and childhood outcomes of the offspring. Different types of parental consanguinity were also included in our analysis to provide the stratified risks according to the degree of inbreeding. Collection of socio-economic characteristics was also comprehensive. The same composition of ethnicity in both the consanguineous and control groups further minimised the socio-economic confounding effects in the analysis. Another limitation is that the genetic data were often incomplete or not up-todate for the studied cases, which were recorded from 2007 to 2016.

It is recommended that a territory-wide prospective study is conducted on consanguineous couples to further delineate their healthcare needs in Hong Kong.

\section{Conclusions}

Identification of consanguineous couples is essential to ensure appropriate referral for preconception or prenatal counselling and diagnosis. Our study showed the majority of consanguineous unions in Hong Kong are of Pakistani ethnicity. International studies have reported that in addition to recessive genetic diseases, offspring of consanguineous unions have higher incidences of non-genetically confirmed structural abnormalities, developmental delay, and autism spectrum disorders. The present study confirms this in the Hong Kong population. Information on the increased risks associated with parental consanguinity should be included in genetic counselling for consanguineous couples, so that they can make informed decisions.

\section{Author contributions}

All authors had full access to the data, contributed to the study, approved the final version for publication, and take responsibility for its accuracy and integrity. 
Concept or design of the study: All authors.

Acquisition of data: KH Siong.

Analysis or interpretation of data: KH Siong, TY Leung.

Drafting of the manuscript: KH Siong, TY Leung.

Critical revision for important intellectual content: All authors.

\section{Conflicts of interest}

The authors have no conflicts of interest to disclose.

\section{Funding/support}

This research received no specific grant from any funding agency in the public, commercial, or not-for-profit sectors.

\section{Ethics approval}

Ethics approval was obtained from New Territories West Cluster Clinical Research Ethics Committee (Ref NTWC/ CREC/18012).

\section{References}

1. Bittles A. Consanguinity and its relevance to clinical genetics. Clin Genet 2001;60:89-98.

2. Consang.net. Global prevalence of consanguinity. Available from: http://www.consang.net/index.php/ Global_prevalence. Accessed 1 Apr 2018.

3. Posch A, Springer S, Langer M, Blaicher W, Streubel B, Schmid M. Prenatal genetic counseling and consanguinity. Prenat Diagn 2012;32:1133-8.

4. Becker R, Keller T, Wegner RD, et al. Consanguinity and pregnancy outcomes in a multi-ethnic, metropolitan European population. Prenat Diagn 2015;35:81-9.

5. Kapurubandara S, Melov S, Shalou E, Alahakoon I. Consanguinity and associated perinatal outcomes, including stillbirth. Aust N Z J Obstet Gynaecol 2016;56:599-604.

6. Hong Kong SAR Government. Hong Kong 2016 Population By-census. Available from: https://www.bycensus2016.gov. hk/en/bc-articles.html. Accessed 1 Apr 2018.

7. Barrett P. A review of consanguinity in Ireland-estimation of frequency and approaches to mitigate risks. Ir J Med Sci 2016;185:17-28.

8. Teeuw ME, Hagelaar A, ten Kate LP, Cornel MC, Henneman L. Challenges in the care for consanguineous couples: an exploratory interview study among general practitioners and midwives. BMC Fam Pract 2012;13:105.

9. Aalfs CM, Smets EM, de Haes HC, Leschot NJ. Referral for genetic counselling during pregnancy: limited alertness and awareness about genetic risk factors among GPs. Fam Pract 2003;20:135-41.

10. Bennett RL, Hudgins L, Smith CO, Motulsky AG. Inconsistencies in genetic counseling and screening for consanguineous couples and their offspring: the need for practice guidelines. Genet Med 1999;1:286-92.

11. Joseph N, Pavan KK, Ganapathi K, Apoorva P, Sharma $\mathrm{P}$, Jhamb JA. Health awareness and consequences of consanguineous marriages: a community-based study. J Prim Care Community Health 2015;6:121-7.

12. Jaber L, Romano O, Halpern GJ, Livne I, Green $M$, Shohat T. Awareness about problems associated with consanguineous marriages: survey among Israeli Arab adolescents. J Adolesc Health 2005;36:530.
13. Teeuw ME, Loukili G, Bartels EA, ten Kate LP, Cornel MC, Henneman L. Consanguineous marriage and reproductive risk: attitudes and understanding of ethnic groups practising consanguinity in Western society. Eur J Hum Genet 2014;22:452-7.

14. Khalil A, Rezende J, Akolekar R, Syngelaki A, Nicolaides KH. Maternal racial origin and adverse pregnancy outcome: a cohort study. Ultrasound Obstet Gynecol 2013;41:278-85.

15. Mumtaz G, Nassar AH, Mahfoud Z, et al. Consanguinity: a risk factor for preterm birth at less than 33 weeks' gestation. Am J Epidemiol 2010;172:1424-30.

16. Islam MM. Effects of consanguineous marriage on reproductive behaviour, adverse pregnancy outcomes and offspring mortality in Oman. Ann Hum Biol 2013;40:24355.

17. Hosseini-Chavoshi M, Abbasi-Shavazi MJ, Bittles AH. Consanguineous marriage, reproductive behaviour and postnatal mortality in contemporary Iran. Hum Hered 2014;77:16-25.

18. Bhopal RS, Petherick ES, Wright J, Small N. Potential social, economic and general health benefits of consanguineous marriage: results from the Born in Bradford cohort study. Eur J Public Health 2014;24:862-9.

19. Teebi AS, El-Shanti HI. Consanguinity: implications for practice, research, and policy. Lancet 2006;367:970-1.

20. Fareed M, Afzal M. Genetics of consanguinity and inbreeding in health and disease. Ann Hum Biol 2017;44:99-107.

21. Committee on Genetics. Committee Opinion No. 690: Carrier screening in the age of genomic medicine. Obstet Gynecol 2017;129:e35-40.

22. Stoll C, Alembik Y, Roth MP, Dott B. Parental consanguinity as a cause for increased incidence of births defects in a study of 238,942 consecutive births. Ann Genet 1999;42:133-9.

23. Sheridan E, Wright J, Small N, et al. Risk factors for congenital anomaly in a multiethnic birth cohort: an analysis of the born in Bradford study. Lancet 2013;382:1350-9.

24. Nabulsi MM, Tamim H, Sabbagh M, Obeid MY, Yunis KA, Bitar FF. Parental consanguinity and congenital heart malformations in a developing country. Am J Med Genet A 2003;116:342-7.

25. Becker SM, Al Halees Z, Molina C, Paterson RM. Consanguinity and congenital heart disease in Saudi Arabia. Am J Med Genet 2001;99:8-13.

26. Yunis K, Mumtaz G, Bitar F, et al. Consanguineous marriage and congenital heart defects: a case-control study in the neonatal period. Am J Med Genet A 2006;140:152430 .

27. Abbas HA, Yunis K. The effect of consanguinity on neonatal outcomes and health. Hum Hered 2014;77:87-92.

28. Berends AL, Steegers EA, Isaacs A, et al. Familial aggregation of preeclampsia and intrauterine growth restriction in a genetically isolated population in The Netherlands. Eur J Hum Genet 2008;16:1437-42.

29. Sezik M, Ozkaya O, Sezik HT, Yapar EG, Kaya H. Does marriage between first cousins have any predictive value for maternal and perinatal outcomes in pre-eclampsia? J Obstet Gynaecol Res 2006;32:475-81.

30. Poorolajal J, Ameri P, Soltanian A, Bahrami M. Effect of consanguinity on low birth weight: a meta-analysis. Arch Iran Med 2017;20:178-84. 